\title{
Impacto de la cercanía, toque afectivo y satisfacción con el apoyo de la pareja en los comportamientos saludables
}

\section{Impact of closeness, affective touch and satisfaction with the couple's support on healthy behaviors}
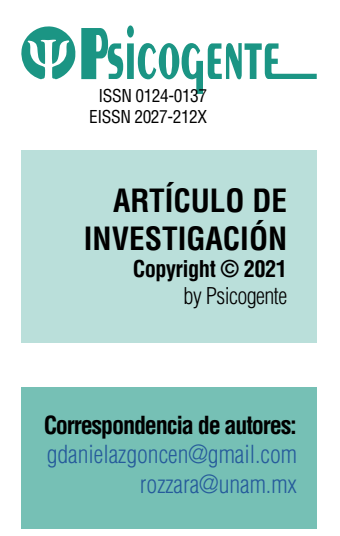

Recibido: 05-02-2020 Aceptado: 08-10-2020 Publicado: 01-01-2021
Georgina D. Zepeda-Goncen (iD) - Rozzana Sánchez-Aragón (iD

Universidad Nacional Autónoma de México, Ciudad de México

\section{RESUMEN}

Introducción: La pareja tiene efectos físicos y psicológicos sumamente positivos, especialmente cuando hay cercanía, debido al bienestar experimentado, toque afectivo, que enriquece la expresión de comprensión y satisfacción con el apoyo de la pareja, pues habrá mayor motivación para realizar comportamientos saludables.

Objetivo: Identificar la magnitud, examinar las diferencias y conocer el grado de asociación de la cercanía, toque afectivo y satisfacción con el apoyo recibido por parte de la pareja en el empleo de comportamientos saludables.

Método: Se trabajó con una muestra no probabilística de 194 hombres y 194 mujeres mexicanos integrantes de una relación de pareja heterosexual (edad $M=37$ ), con escolaridad mínima de secundaria. Se aplicó una batería compuesta por instrumentos de medición psicológica validados para población mexicana y se realizó una serie de análisis de varianza con pruebas a posteriori de Scheffé y correlaciones producto momento de Pearson.

Resultados: De los resultados se destaca que los grupos de mayor toque afectivo y satisfacción con el apoyo recibido emplean mayores comportamientos saludables de actividad y relajación. Por otra parte, para las mujeres, la cercanía se relaciona con mayores conductas de Actividad-relajación (0,168* según Pearson). Para los hombres, mayor satisfacción con el apoyo de su pareja propiciará que realicen mayores conductas de cuidado (0,157* según Pearson).

Conclusión: Formar parte de una relación de pareja cercana, afectiva y donde el apoyo es satisfactorio crea un espacio para tener mejor calidad de vida y salud, por su influencia sobre actitudes, significados y conocimientos que determinan la adopción de conductas saludables.

$\overline{\text { Palabras clave: cercanía afectiva; toque afectivo; apoyo social; comportamientos saludables; relación }}$ de pareja.

\section{ABSTRACT}

Introduction: The couple has extremely positive physical and psychological effects, especially when there is closeness, due to the experienced well-being, affective touch, which enriches the expression of compression and satisfaction with the support of the couple, since there will be greater motivation to perform healthy behaviors.

Objective: Identify the magnitude, examine the differences and know the degree of association of closeness, affective touch and satisfaction with the support received from the couple in the use of healthy behaviors.

Method: We worked with a non-probability sample of 194 Mexican men and 194 Mexican women who were members of a heterosexual couple relationship (age $M=37$ ), with minimum middle school studies. A battery composed of validated psychological measurement instruments for the Mexican population was applied and a series of analysis of variance was performed with Scheffe's posterior tests and Pearson's product-moment correlations.

Results: From the results, it is highlighted that the groups with the greatest affective touch and satis- 
faction with the support received employ greater healthy behaviors of activity and relaxation. On the other hand, for women, closeness is related to greater Activity-relaxation behaviors $\left(0,168^{*}\right.$ according to Pearson). For men, greater satisfaction with the support of their partner will lead to more caring behaviors (0,157* according to Pearson)

Conclusion: Being part of a close, affective and supportive couple relationship creates a space to have better quality of life and health, due to its influence on attitudes, meanings and knowledge that determine the adoption of healthy behaviors.

Key words: affective closeness; affective touch; social support; healthy behaviors; couple relationships.

\section{INTRODUCCIÓN}

En la experiencia amorosa se vive una transformación producida por el enamoramiento, en donde las personas van a percibir, en diferentes medidas, que sus fronteras individuales se ablandan y que se entra en un estado de fusión con la pareja, experimentando sensaciones de entrega total, plenitud, goce, éxtasis, entre otras; mismas que abren la posibilidad a una interacción más fuerte y una nueva identidad producto de la vinculación amorosa que constituye a la pareja. Este fenómeno ha sido denominado cercanía (Villegas Besora, 2006) y representa un concepto imprescindible para explicar la teoría e investigación de las relaciones interpersonales, pues impacta a una amplia gama de fenómenos relacionales que van desde la autorrevelación hasta la resolución de conflictos (Dibble, Levine, \& Park, 2011).

La cercanía es uno de los componentes más importantes para los integrantes de la diada puesto que, con esta, se logra cubrir la necesidad de interacción constante y comprometida de los miembros (Alzugaray \& García, 2015) y puede ser definida por su naturaleza como la conexión social de intercambio de afecto y la percepción de intimidad entre quienes conforman la relación (Ramos Salazar, 2015). Este constructo hace alusión a cuando un miembro de la pareja incluye al otro en su yo, dando pie a que la cercanía refiera el grado de superposición del yo con el otro y a la interdependencia en términos cognoscitivos, afectivos y conductuales (Aron, Aron, \& Smollan, 1992).

La cercanía implica sentimientos que producen que la relación se perciba como profunda, estrecha, recíproca, entregada, dependiente y generará que los integrantes de la pareja describan de forma positiva su interacción, fusión y permanencia en la misma y, en consecuencia, pueden estar más satisfechos (Villegas Besora, 2006; Sánchez-Aragón, 2019). La distancia entre los miembros de la diada es un proceso que implica distintos grados de proximidad física, autodivulgación, interacción emocional y cantidad de tiempo empleado juntos o separados y ha sido visualizado como una "danza" en la que ambos se sentirán en algunos periodos más cercanos que en otros, tal como sucede al bailar, generando una dinámica en la que ambos integrantes 
aprenderán a leer las necesidades afectivas del otro y perfeccionarán los patrones de cambio y estabilidad, cercanía y alejamiento, que se mantendrá gracias a un sistema implícito que les permite funcionar adecuadamente y que estará presente en todos los momentos y contextos de la relación (Rosenblatt \& Barner, 2006).

En otras palabras, puede variar significativamente a lo largo de un continuo de proximidad que crecerá con la fuerza del vínculo emocional experimentado por los socios románticos, el grado de conocimiento de la otra persona y su interdependencia afectiva, cognitiva y conductual (Berscheid, Snyder, \& Omoto, 1989; Dibble, Levine, \& Park, 2011).

La cercanía representa un factor tan importante que su ausencia es una de las razones más comunes para que las personas asistan a psicoterapia, refiriendo pérdida de amor y conexión con la relación, misma que les produce frustración y desesperanza para recobrar la intimidad perdida (Fife \& Weeks, 2010).

Para lograr la cercanía y con ella sus beneficios, la afectividad manifestada a través del contacto o toque afectivo resulta ser uno de sus ingredientes centrales. Este contacto implica gestos terapéuticos que van cargados de significados como el amor, la solidaridad, cariño, comprensión, aceptación, bondad, dulzura, confianza, calidez, reconocimiento y otros sentimientos que liberan diferentes neurotransmisores que ayudan a mejorar la calidad de vida, entre ellos la oxitocina, que produce relajación y ayuda a fortalecer los vínculos, teniendo implicaciones incalculables (Morales, 2016; Da Silva, 2014). Desde la perspectiva de Pozos-Gutiérrez, Rivera-Aragón, Reyes-Lagunes, y López-Parra (2013) el toque afectivo involucra: arrumacos, intimidad, apapachos, ternura, abrazos, caricias y afecto, características que son necesarias para que exista felicidad en la pareja y adecuado mantenimiento de la relación.

El toque afectivo es el lenguaje del cuerpo, que en muchas ocasiones habla más que de lo que se puede expresar con las palabras, es un diálogo físico que puede transmitir un abanico de mensajes desde relajación, apoyo, alerta, amor, complicidad, etc. (Da Silva, 2014). Por ello, esta variable impacta de diversas maneras, pues las caricias generan evolución personal y madurez emocional, ayudan a mejorar la autoestima, tener mayor seguridad y relacionarse de mejor manera con el resto del mundo, mientras que los abrazos y el contacto físico son sumamente importantes para el desenvolvimiento adecuado en diferentes contextos sociales y mejor desarrollo emocional (Morales, 2016), puesto que, además, este tacto tiene propiedades especí- 
ficas para reducir el estrés, así como propiedades preventivas para evitarlo, esto tanto a nivel fisiológico como subjetivo (Debrot, Schoebi, Perrez, \& Horn, 2013).

El papel de este contacto físico interpersonal es tan crítico para el desarrollo humano, de salud, comunicación y vínculo social, que en la actualidad se ha mostrado un creciente interés en las investigaciones en psicología, desde los enfoques neurocientíficos hasta sociales. Dentro de los hallazgos más recientes se ha descubierto que, durante la interacción, el nivel fisiológico puede llegar a tener más importancia incluso que las conductas o expresiones verbales, especialmente cuando se trata de vínculos con fuertes lazos emocionales como la pareja, en la que se experimenta un afecto intenso (Chatel-Goldman, Conged, Jutten, \& Schwartz, 2014).

Es así que la diada, por medio de la cercanía y el toque afectivo, va a desarrollar capacidades emocionales para amortiguar y protegerse de los efectos del estrés cotidiano, pero, además, va a generar múltiples beneficios para la salud, calidad de vida y bienestar de sus integrantes debido a los recursos que se desprenden del apoyo recibido de la pareja (Domínguez, 2012). Dado que esta relación es la principal fuente de apoyo de la adultez en vista de la especial motivación a responder a las necesidades del otro (Debrot, 2012), la mayor influencia al compartir el espacio habitable, tiempo, finanzas e intimidad física, en donde el compañero romántico va a ayudar a hacer frente a las demandas del medio y aportar soluciones no solo en eventos difíciles de afrontar, sino en la vida diaria, que es más llevadera y alentadora si la persona tiene más confianza acerca de su capacidad para manejar la situación al saber que su compañero de apoyo estará presente (Galione, 2016).

El apoyo de parte de la pareja se ha concebido como la creencia de ser cuidado, amado, estimado, valorado y forma parte de una red de comunicación y cuidados mutuos (Ramos Salazar, 2015) y al respecto, Fernández-Peña (2005) señala que, de forma especial, las personas afectadas por problemas emocionales, así como quienes hacen frente al curso de enfermedades e incapacidad, eligen a la pareja como primordial apoyo dentro del entorno próximo para la resolución activa de los problemas y para el mantenimiento de la salud mental y física.

Matud, Cabelleira, López, Marrero e Ibáñez (2002) explican que, algunas hipótesis apuntan a que el apoyo de la pareja surte un efecto directo en la salud, mientras que otros planteamientos sugieren un efecto indirecto o mediador. Este último se basa en la influencia de parte de la pareja en 
actitudes y comportamientos saludables, que van a tener efectos en la salud y la longevidad. Es decir, una de las principales explicaciones para entender el vínculo entre el matrimonio y la salud, deriva de los beneficios que las personas casadas obtienen a través del apoyo social que reciben de sus cónyuges, actuando en una variedad de mecanismos como promover un estilo de vida más saludable (Tucker \& Anders, 2001).

En el estudio del apoyo que proviene de la pareja, se ha resaltado la evaluación cognitiva que la persona hace del mismo, por lo que es de suma importancia considerar la dimensión subjetiva, es decir, la forma en que es percibido este apoyo y la satisfacción que se siente respecto al mismo, ya que esta valoración es la que se considera en mayor medida, promotora de salud. La importancia de hacer esta distinción radica en que algunas personas reciben apoyo, pero no lo perciben y no hacerlo produce que ese apoyo disponible no pueda ser utilizado (Fernández-Peña, 2005).

Otra de las dimensiones que debe considerarse con respecto a la satisfacción del apoyo que se recibe de parte de la pareja y que se vincula con los efectos benéficos de esta relación para la salud de sus integrantes es la reciprocidad, ya que en la medida en que se evalúa que los recursos intercambiados son equitativos y, en términos generales, la relación parece recíproca, se promueve en mayor medida la adopción de comportamientos más saludables. Por el contrario, percibir que el apoyo recibido no es proporcional, semejante y satisfactorio en proporción al que se brinda, se relaciona estrechamente con insatisfacción y problemas de salud mental (Fernández-Peña, 2005).

Walker, Lucier-Geer, Mancini, Ferrato y Ross (2016) postulan que, la influencia de relaciones cercanas como la familia y la pareja facilitan un entorno y recursos que promueven mayor calidad de vida. En el caso específico de la pareja, cuando la relación es estable, cercana, satisfactoria y una fuente de apoyo, propiciará que sus integrantes tengan mejor salud mental y recursos psicológicos y exhiban en mayor medida comportamientos saludables.

Entonces, cuando se siente satisfacción con el apoyo recibido por parte de la pareja, habrá mayor motivación para la realización de comportamientos saludables. Estos comportamientos y estilos de vida saludables pueden definirse como la participación de un estilo que mejora la calidad de vida que se asocia con una menor morbilidad y menor riesgo de mortalidad (Tucker \& Anders, 2001) y en los cuales se incluyen: Adquirir y mantener mejores hábitos alimenticios y de sueño, realizar más actividad física, menor exposición a 
conductas de riesgo, uso más apropiado de cigarro, alcohol y drogas, mayor adherencia a prescripciones médicas y seguimiento de cuidados físicos.

Estas conductas van a presentarse como mediadoras de los efectos de la conexión o desconexión de la salud y el bienestar que se puede generar de las relaciones interpersonales (Dunkel, 2017), teniendo que, cuando se habla de personas que participan de relaciones de pareja positivas, como aquellas que son cercanas y donde se expresan y manifiestan los sentimientos de amor y cariño, se contará con más recursos psicológicos que sirven para participar de hábitos de sueño y de alimentación más saludables. Por el contrario, los sistemas familiares estresantes parecen reducir la disponibilidad de recursos psicológicos y, a su vez, aumentar el riesgo de emplear comportamientos perjudiciales para la calidad de vida (Walker et al., 2016).

Al respecto, la literatura ha arrojado un cuerpo de evidencia que demuestra además que las personas tienden a exhibir comportamientos de salud similares al de quienes les rodean, asociación que se vuelve más fuerte en la medida en que el vínculo es más cercano y para el que la pareja representa una de las relaciones más influyentes para, por ejemplo, dejar de fumar (Jackson, Steptoe, \& Wardle, 2015).

calidad de la relación, que aumenta en la medida en que una pareja es cercana, cariñosa y se apoya, también va a regular la forma en que se emplean conductas relacionadas con la salud, como la actividad física realizada versus un estilo de vida sedentario, con conductas tales como destinar muchas horas a ver televisión, videojuegos o consumo de sustancias adictivas (Walker et al., 2016).

En torno a esto, autores como Jackson, Steptoe, y Wardle (2015) explican que esto se da por los sentimientos de responsabilidad, compromiso y obligación hacia la persona significativa, que provocan la participación en un estilo de vida más saludable. Por otra parte, la literatura ha dejado en evidencia que, en la medida en que se empleen estrategias positivas, como motivar o recompensar, para intentar mejorar los hábitos de la pareja, habrá mayor eficacia, mientras que cuando se utilicen estrategias negativas tales como recriminar o prohibir, se presentará una tendencia por ignorar al cónyuge, hacer lo contrario u ocultar los comportamientos poco saludables (Tucker \& Anders, 2001).

La forma en que se percibe la relación, en términos de qué tan cercana es, el toque afectivo, el nivel de satisfacción con el vínculo y el apoyo social 
brindado de parte de la pareja puede proporcionar motivación para la realización de estos comportamientos saludables (Galione, 2016). Lo anterior se respalda desde una perspectiva integral, para la cual, los procesos de salud-enfermedad deben ser vistos en consideración de la dimensión social de la salud, en donde el grado de integración o aislamiento al medio social se asocia estrechamente con el grado de bienestar y salud que se posee (Fernández-Peña, 2005). Con esto se concibe a la salud como un proceso que necesariamente incluye el identificar, adquirir, mantener y desarrollar comportamientos saludables (Cortés-Vázquez, Ramírez-Guerrero, OlveraMéndez, \& Arriaga-Abad, 2009) motivados por contar con relaciones interpersonales cercanas, positivas e influyentes y que traen consigo beneficios provenientes de la participación de un vínculo que fomenta la adopción de hábitos y estilos de vida benéficos (Dunkel, 2017).

Bajo este marco, el problema de investigación abordado se centra en estudiar la relación entre la cercanía y el toque afectivo, así como la satisfacción con el apoyo que brinda la pareja en el empleo de comportamientos saludables, para explorar cómo la percepción y evaluación de las personas sobre estos constructos representa una vía que conecta a la relación de pareja con la salud de sus integrantes, configurando un fenómeno pertinente para estudiarse desde el campo y la metodología de la Psicología Social cuantitativa.

Partiendo de esta problematización, los objetivos de esta investigación fueron: 1) Identificar la magnitud de cada una de las variables estudiadas a partir de sus medias, 2) Examinar las diferencias en el despliegue de comportamientos saludables para explorar la influencia del grado de cercanía, toque afectivo del otro y la satisfacción con el apoyo recibido por parte de la pareja y 3 ) Conocer el grado de asociación de las mismas variables sobre los comportamientos saludables en parejas mexicanas.

\section{MÉTODO}

\subsection{Participantes}

Se trabajó con una muestra no probabilística propositiva (Kerlinger \& Lee, 2002), cuyos criterios de inclusión consistieron en que las y los participantes fueran mayores de edad (18 años), formaran parte de una relación de pareja heterosexual y, al momento de contestar los instrumentos, hubieran cohabitado con su pareja por al menos un año. Se contó con la participación de 388 residentes de la Ciudad de México (194 hombres y 194 mujeres), cuyas edades oscilaron entre los 18 y 64 años $(M=37, D E=11,34)$. En cuanto a la escolaridad, 74 participantes tenían estudios de secundaria, 132 de prepara- 
toria y 182 de licenciatura. Del total de participantes, 180 eran casados y 208 señalaron vivir en unión libre, el tiempo medio de cohabitación fue de 12 años $(D E=9,97)$. Finalmente, 188 reportaron no tener hijos y 200 sí $(\mathrm{Mo}=2)$.

\subsection{Instrumentos}

Para dar cumplimiento a los objetivos antes mencionados, se aplicó una batería compuesta por los siguientes instrumentos:

\subsubsection{Escala de Inclusión del Otro en el Yo (Aron et al., 1992; Sánchez-} Aragón, 1995)

Instrumento que evalúa directamente el sentir de la interconectividad interpersonal en la que los participantes responden seleccionando la imagen que mejor describa su relación por grados de traslape progresivo lineal entre el yo y el otro. La prueba original fue adaptada y validada en población mexicana, conservando su estructura original, misma que consiste en un reactivo pictórico con siete opciones de respuesta en diagramas de Venn que se van traslapando y miden el grado de cercanía con base de la interrelación entre los miembros de la pareja.

\subsubsection{Escala de Toque Afectivo (Debrot et al., 2013; Sánchez-Aragón} 2018)

Instrumento que se concentra en la evaluación y medición de las expresiones de toque que denotan afecto y amor romántico. Consta de siete afirmaciones que reflejan diferentes expresiones de ternura y afecto de parte del compañero romántico con un formato de respuesta tipo Likert de 5 puntos que indican grados de frecuencia ( $1=$ Nunca y $5=$ Siempre). Ejemplos de los ítems que le componen son los siguientes: "Frecuencia con la que toma la mano de su pareja" y "Frecuencia con la que se sienta o acuesta junto a su pareja durante la lectura, ver televisión o realizar alguna actividad de ocio". Cabe señalar que en el artículo original no se reportan datos de validación, pero en la validación en México realizada con 424 parejas heterosexuales se obtuvo un alpha total de 0,86 y a partir de un análisis exploratorio y confirmatorio, se confirmó su unidimensionalidad.

\subsubsection{Escala de Satisfacción con el Apoyo Recibido de la Pareja (ESAReP)}

(Sánchez-Aragón, 2019)

Instrumento que evalúa el grado de satisfacción que una persona experimenta con respecto al apoyo social que recibe de parte de su pareja, validado en parejas mexicanas. Consta de 30 reactivos con formato de respuesta tipo Likert de 5 puntos en donde $1=$ "Totalmente insatisfecho" y 5 = "Muy satis- 
fecho" distribuidos en dos factores: 1) En la cotidianidad: Con reactivos como: "con la ayuda que me da mi pareja para sentirme mejor cuando estoy muy irritable" y "Cuando mi pareja me consuela si estoy muy enojado"; y 2) En la gravedad: Con reactivos como: "que poseen coeficientes Alfa de Cronbach de 0,975 a 0,977 y explican $61,128 \%$ de la varianza.

\subsection{4. Índice de Comportamientos Saludables (Santiago-Silva, 2020)}

Medida diseñada para evaluar el empleo de conductas que se relacionan con el mejoramiento o el detrimento de la salud en adultos mexicanos y se conforma por 18 ítems con formato de respuesta tipo Likert que indican 5 grados de frecuencia, distribuidos en 3 factores y un indicador con valores propios superiores a 1, que explican el 45,459 \% de varianza, cuyos coeficientes de confiabilidad Alfa de Cronbach oscilaron de 0,627 a 0,686. Los factores e indicador están diseñados para evaluar: Descuido $(\alpha=0,628)$ : Con reactivos como "Cuando tengo hambre, no me detengo a ver la procedencia de mis alimentos" y "Uso el teléfono celular mientras manejo o camino". Actividad física y relajación $(\alpha=0,627)$ : Se compone de ítems como "Sigo una rutina de ejercicios todos los días" y "Suelo elegir usar las escaleras, aunque haya un elevador disponible". Prevención de Adicciones $(\alpha=0,685)$ : Ejemplo de sus reactivos "Evito consumir cualquier tipo de droga" y "Trato de no consumir bebidas alcohólicas". El último factor es Cuidado $(\alpha=0,686)$ : Con afirmaciones como "Procuro caminar con precaución cuando el piso está mojado" y "Soy cuidadoso(a) al realizar actividades que involucren cuchillos o fuego".

\subsection{Procedimiento}

Los cuestionarios fueron aplicados por psicólogas con la preparación técnica y ética para conducirse con las personas, solicitar su participación voluntaria, confidencial y anónima, explicando el manejo de las respuestas que proporcionaran, así como el fin estadístico de sus datos.

Estas profesionistas acudieron a lugares públicos donde se encontraron a los participantes (escuelas, oficinas, plazas comerciales, casas, etc.), para que contestaran en forma voluntaria a la batería en cuestión. Ante cualquier duda que se presentara durante la aplicación, se dio respuesta inmediata.

Cada una de las personas que participó en la presente investigación, cumplió con los siguientes criterios de inclusión: Ser mayor de edad, tener una escolaridad mínima de secundaria, tener una relación de pareja heterosexual y haber cohabitado con esta persona por lo menos un año antes de parti- 
cipar en el estudio. En el caso de no cumplir alguno de estos requisitos, no formaron parte de la muestra.

\subsection{Análisis de datos}

Una vez completada la aplicación, los instrumentos fueron capturados en una base de datos del paquete estadístico informático IBM SPSS. En el mismo Software fueron procesados y analizados los datos conforme a los objetivos de la investigación en el siguiente orden: Primero, se corrió una serie de análisis de estadísticos descriptivos para apuntar las medias con su respectiva desviación estándar para cada variable interventora.

Posteriormente, se analizó la distribución de las respuestas de todos los participantes con el fin de conformar tres grupos que responden al grado de los constructos estudiados en términos de bajo, medio y alto para la cercanía, toque afectivo y satisfacción con el apoyo recibido por parte de la pareja, de tal forma que los grupos resultaran equivalentes en número. Con base en estos grupos se realizaron una serie de análisis de varianza de una vía con pruebas a posteriori de Scheffé para así examinar las posibles diferencias en los comportamientos saludables.

Por último, respondiendo al tercer objetivo planteado, se ejecutó una serie de correlaciones producto-momento de Pearson para conocer el grado de asociación de la cercanía, el toque afectivo y la satisfacción con el apoyo de la pareja en los comportamientos saludables de las mujeres y hombres que conforman las parejas de la muestra.

\section{RESULTADOS}

Al identificar la magnitud de las variables estudiadas en términos de sus medias, los participantes reportan una alta cercanía $(M=4, D E=1,82)$, al tener un importante grado de traslape entre el yo y el otro de acuerdo con los diagramas de Venn del instrumento aplicado. En cuanto al nivel de toque afectivo, se reportan frecuencias que indican que las parejas de los participantes realizan conductas, tales como tomar la mano, dar masajes en el cuello y la espalda y abrazar varias veces a la semana $(M=3, D E=1,13)$.

La satisfacción con el apoyo social recibido es ligeramente más alta cuando se trata del apoyo reportado en el día a día $(M=4,29, D E=0,766)$, mientras que en situaciones de gravedad tiene una media ligeramente más baja de 4,11 ( $D E=0,759)$. Sin embargo, en ambas condiciones, estos resultados indican que los participantes se encuentran de satisfechos a muy satisfechos con la confianza que su pareja les da para ser totalmente ellos mismos (Apoyo en lo 
cotidiano) y con el apoyo que les daría su pareja si algún miembro cercano de su familia muriera (Apoyo en la gravedad).

Para los comportamientos saludables, la muestra aquí considerada reporta casi nunca usar el teléfono mientras camina o maneja (Descuido, $M=2,45$, $D E=0,757)$ ), suelen elegir usar las escaleras, aunque haya un elevador disponible (Actividad y relajación, $M=3,33$; $D E=0,776$ ), tratan de no consumir bebidas alcohólicas (Prevención de adicciones, $M=3,54, \mathrm{DE}=1,19$ ) y casi siempre procuran caminar con precaución (Cuidado, $M=4,30,0,786$ ) (ver Tabla 1).

Tabla 1.

Medias y Desviaciones estándar de la cercanía, toque afectivo del otro, satisfacción con el apoyo social recibido y comportamientos saludables

\begin{tabular}{ccc}
\hline FACTOR & M & DE \\
\hline Cercanía & 4,88 & 1,82 \\
Toque Afectivo del otro & 3,36 & 1,13 \\
\hline \multicolumn{2}{c}{ SATISFACCIÓN CON EL APOYO SOCIAL RECIBIDO } \\
\hline Apoyo en lo cotidiano & 4,29 & 0,766 \\
Apoyo en la gravedad & 4,11 & 0,759 \\
\hline \multicolumn{3}{c}{ COMPORTAMIENTOS SALUDABLES } \\
\hline Descuido & 2,45 & 0,757 \\
Actividad y Relajación & 3,33 & 0,776 \\
Prevención de Adicciones & 3,54 & 1,19 \\
Cuidado & 4,30 & 0,786 \\
\hline
\end{tabular}

Se procedió a examinar en qué medida se reportan comportamientos saludables en función de tres niveles de cercanía, toque afectivo de la pareja y apoyo social recibido para poner a prueba el supuesto hipotético que hace referencia a que mayores niveles en estas variables facilitará estilos de vida más saludables.

Para tal propósito, se analizó la distribución de las respuestas y con base en ello se conformaron tres grupos de acuerdo con el grado de cada variable (bajo, medio y alto). A partir de estos grupos se desarrollaron los análisis de varianza con pruebas a posteriori de Scheffé encaminados a examinar las posibles diferencias en las variables bajo estudio.

En el caso de la cercanía, los grupos representaron un 32,8 \% para el nivel bajo, 33,2 \% para cercanía media y $34 \%$ para alta, sin embargo, el ANOVA no arrojó diferencias estadísticamente significativas en la frecuencia de comportamientos saludables por esta variable (ver Tabla 2). 
Los grupos que se desprenden de la distribución del toque afectivo del otro representan las siguientes proporciones: Bajo $24 \%$, medio $36 \%$ y alto $40 \%$. Referente a esta variable, los participantes reportarán mayor cantidad de comportamientos saludables de actividad y relajación, tales como buscar momentos en el día para relajarse y descansar, en la medida en la que reciban de parte de su pareja caricias y abrazos, seguidos por el grupo de toque afectivo medio, finalmente, el grupo de bajo toque afectivo será quien menos reporte emplear este tipo de comportamientos saludables (ver Tabla 2).

Para el apoyo social de parte de la pareja, un 28,2 \% reportó baja satisfacción, $35,3 \%$ media y $36,5 \%$ alta. Con estos grupos de comparación fue posible identificar una diferencia estadísticamente significativa, que con la prueba Scheffé se identificó entre los tres grupos comparados, siendo las personas que en mayor medida saben con certeza que cuentan con la escucha atenta de su pareja (alta satisfacción con el apoyo social de la pareja), quienes más reportarán comportamientos saludables de actividad y relajación como seguir una rutina de ejercicios de acondicionamiento físico todos los días, seguidas de aquellos quienes tengan una satisfacción media en la variables en cuestión y por último, aquellos cuya satisfacción sea baja con el apoyo que les viene de sus parejas (ver Tabla 2).

Tabla 2.

Diferencias en los factores de los comportamientos saludables por el grado de cercanía, toque afectivo del otro y satisfacción con el apoyo social recibido

\begin{tabular}{cccccc}
\hline FACTOR & BAJO & MEDIO & ALTO & F & P \\
\hline \multicolumn{7}{c}{ CERCANÍA } \\
\hline Descuido & 2,46 & 2,47 & 2,39 & $(2 / 0,448) ; 0,389$ & 0,678 \\
Actividad y Relajación & 3,28 & 3,32 & 3,43 & $(2 / 0,588) ; 0,3977$ & 0,377 \\
Prevención de Adicciones & 3,58 & 3,49 & 3,54 & $(2 / 0,370) ; 0,259$ & 0,772 \\
Cuidado & 4,20 & 4,31 & 4,42 & $(2 / 1,514) ; 2,46$ & 0,086 \\
& \multicolumn{7}{c}{ TOQUE AFECTIVO DEL OTRO } & & 0,149 \\
Descuido & 2,36 & 2,54 & 2,44 & $(2 / 1,098) ; 1,91$ & 0,019 \\
Actividad y Relajación & 3,18 & 3,36 & 3,45 & $(2 / 2,40) ; 4,02$ & 0,609 \\
Prevención de Adicciones & 3,60 & 3,57 & 3,46 & $(2 / 0,710) ; 0,497$ & 0,360 \\
Cuidado & 4,21 & 4,32 & 4,35 & $(2 / 0,635) ; 1,02$ & \\
& SATISFACCIÓN CON EL APOYO SOCIAL RECIBIDO & $(2 / 0,559) ; 0,974$ & 0,378 \\
Descuido & 2,45 & 2,53 & 2,40 & $(2 / 5,05) ; 8,80$ & 0,000 \\
Actividad y Relajación & 3,10 & 3,38 & 3,48 & $(2 / 1,13) ; 0,804$ & 0,449 \\
Prevención de Adicciones & 3,46 & 3,50 & 3,63 & $(2 / 1,71) ; 2,70$ & 0,068 \\
Cuidado & 4,16 & 4,33 & 4,37 & & \\
\hline
\end{tabular}


Para dar cumplimiento al último objetivo, se realizaron algunos análisis de correlación producto momento de Pearson para mujeres y hombres por separado.

En el caso de ellas se observa que, a mayor percepción de cercanía respecto a su pareja, mayor reporte de conductas de cuidado (revisión de manchas, irritaciones y bultos en alguna parte del cuerpo) y de actividad y relajación, al sentir que dedican más tiempo a la semana para realizar actividades como seguir una rutina de ejercicios (en casa o gimnasio). Asimismo, se observa que, a mayor reporte de toque afectivo de su pareja hacia ellas y más satisfacción en la vida cotidiana, consideran que realizan comportamientos de actividad y relajación como hacer actividades de ocio o recreativas y mayor esfuerzo por encontrar momentos durante el día para relajarse (ver Tabla 3).

Tabla 3.

Correlaciones entre cercanía, toque afectivo del otro, satisfacción con el apoyo social recibido y comportamientos saludables para mujeres.

\begin{tabular}{ccccc}
\hline & DESCUIDO & ACTIVIDAD Y RELAJACIÓN & PREVENCIÓN DE ADICCIONES & CUIDADO \\
\hline Cercanía & - & $0,168^{*}$ & - & $0,206^{* *}$ \\
Toque Afectivo del otro & - & $0,250^{* *}$ & - & - \\
Apoyo en lo cotidiano & - & $0,236^{* *}$ & - \\
Apoyo en la gravedad & - & - & - & - \\
\hline
\end{tabular}

Por su parte, en los hombres se encontró que, en la medida en que ellos reportan más toque afectivo recibido por parte de sus parejas (como masajes en el cuello o espalda, abrazos, y tener relaciones sexuales con ella) consideran que emplean más comportamientos saludables de actividad y relajación, tales como elegir las escaleras para subir varios pisos, a pesar de que haya un elevador disponible y tomar mínimo dos litros de agua al día. Aunado a ello, se notó que en la medida en que se sienten más satisfechos con el apoyo cotidiano por parte de su pareja (pues esta los escucha de una manera abierta y sin criticarlos cuando hablan de sus sentimientos más profundos), reportan la realización de un mayor número de comportamientos de actividad y relajación, así como de cuidado (al procurar en mayor medida caminar con precaución si el piso esta mojado y/o resbaloso). Finalmente, a más satisfacción con el apoyo recibido por parte de la pareja ante situaciones de gravedad, los participantes reportan más conductas de cuidado (ver Tabla 4). 
Tabla 4.

Correlaciones entre cercanía, toque afectivo del otro, apoyo social y comportamientos saludables para hombres.

\begin{tabular}{ccccc}
\hline & DESCUIDO & ACTIVIDAD Y RELAJACIÓN & PREVENCIÓN DE ADICCIONES & CUIDADO \\
\hline Cercanía & - & - & - & - \\
Toque Afectivo del otro & - & $0,234^{* *}$ & - & - \\
Apoyo en lo cotidiano & - & $0,193^{* *}$ & - & $0,157^{*}$ \\
Apoyo en la gravedad & - & - & - & $0,172^{*}$ \\
\hline
\end{tabular}

\section{DISCUSIÓN}

En cuanto al primer objetivo, los resultados permiten identificar la percepción de un alto grado de cercanía y traslape entre los participantes y sus parejas, esta intimidad es uno de los factores más esenciales para que una persona experimente satisfacción, salud, habilidad de adaptación, felicidad, y sentido de vida. Por el contrario, el hecho de que las personas presenten dificultades para establecer cercanía propiciará que en su relación den comportamientos destructivos tales como la infidelidad o el abuso (Fife \& Weeks, 2010).

El nivel de toque afectivo es frecuente según las respuestas de las y los participantes de la muestra, al respecto se debe mencionar que, este diálogo afectivo tiene una capacidad terapéutica de doble vía, pues los beneficios de este contacto son tanto para quien los da, como para quien los recibe y aunque no tiene una connotación directamente sexual, también puede formar parte del repertorio de preámbulos de este tipo de contextos ( $\mathrm{Da}$ Silva, 2014).

La evaluación referente a la satisfacción con el apoyo social recibido de parte de la pareja en sus dos condiciones (cotidianeidad y gravedad) debe interpretarse a la luz de la relevancia de la comunicación como uno de los factores más importantes para que la relación de pareja funcione adecuadamente y es un predictor de la satisfacción, puesto que es a través de la comunicación que la pareja expresa sus necesidades de apoyo, sentimientos, información relevante y se resuelven los conflictos que pueden surgir de no sentir reciprocidad entre el apoyo brindado y el recibido (Carrillo Medina, 2004).

Los comportamientos de descuido casi nunca son reportados, por el contrario, las conductas saludables de actividad y relajación, prevención de adicciones y cuidado son reportadas con frecuencia. Al respecto, Jackson, Steptoe, y Wardle (2015) señalan que, además de la influencia que la pareja tiene sobre el comportamiento de su cónyuge, la explicación de este fenómeno se complementa con la idea documentada que postula que los individuos 
tienden a emparejarse con personas que son similares en estilos de vida y, en conjunto, es posible entender que el estado conyugal es un fuerte predictor de los cambios en el comportamiento relacionado con la salud.

Para el segundo objetivo, se encontraron diferencias en las respuestas que hacen referencia al empleo de comportamientos saludables de actividad y relajación, ya que estos se relacionan con la medida en la que percibe mayor toque afectivo de parte de la pareja. Esto se puede explicar debido a que el estado afectivo de los participantes mejora en función de ser acariciado por el cónyuge, pues este toque es usado como una estrategia de regulación emocional interpersonal no verbal ante el estrés, facilitando que la pareja se relaje de forma natural, aumentando la capacidad de respuesta a este estímulo, haciendo el soporte emocional más beneficioso (Debrot, Schoebi, Perrez, \& Horn, 2013).

Cuando los participantes reportan este mismo tipo de comportamientos saludables, se presentan diferencias respecto al grado de satisfacción con el apoyo social de parte de la pareja, siguiendo un patrón similar en el que las personas con alta satisfacción con este apoyo son quienes reportarán en mayor medida comportamientos saludables de actividad y relajación. Sin embargo, es importante señalar que este resultado no permite esclarecer qué impacto tiene el hecho que una persona, que incluso antes de iniciar su relación ya tenía hábitos alimenticios saludables, por ejemplo, y los mantiene sin cambios durante la relación y bajo qué condiciones y en qué momentos este estilo de vida puede impactar para el cambio en su pareja (Jackson, Steptoe, \& Wardle, 2015), dejando estas interrogantes como sugerencia para encaminar los esfuerzos de futuras investigaciones.

El último interés de esta investigación fue examinar la relación entre la cercanía, toque afectivo y satisfacción con el apoyo recibido por parte de la pareja sobre los comportamientos saludables en hombres y mujeres mexicanas. Para ello se realizaron algunos análisis de correlación que mostraron efectos similares y otros distintos entre los sexos.

Tal como se describió, el primer hallazgo para las mujeres fue la asociación entre el grado de cercanía y el reporte de conductas saludables de cuidado, así como de actividad y relajación. Este resultado es apoyado por la propia noción de cercanía (Aron et al., 1992; Sánchez-Aragón, 1995) que involucra a la interacción continua, frecuente y variada entre la pareja que facilita la interdependencia emocional, cognoscitiva y conductual entre los miembros de la diada. Así, de entre las conductas que realizan de manera frecuente 
y continua (Berscheid et al., 1989) pueden ser aquellas relacionadas con el auto-cuidado (pisos resbalosos y maniobra de cuchillos), la actividad física como subir escaleras, tener rutinas de ejercicio, así como relajarse mediante actividades de ocio y recreativas. Esto puede favorecer la calidad y fuerza del vínculo, así como la cercanía, mejorando el afecto positivo y fortaleciendo la salud mental de la pareja (Prager \& Roberts, 2004 citado en Debrot et al., 2013). En correspondencia con este hallazgo, Skoyen, Rentscher y Butler (2018) encontraron que, en la medida en que las mujeres describen su relación de pareja, como próxima, amorosa y de calidad, su índice de masa corporal tiende a mantenerse en equilibrio y ser moderado por comportamientos más saludables y ser más bajo dentro de parámetros adecuados en comparación con mujeres de relaciones que no eran descritas en los términos anteriores. Hay que señalar, que en el contexto de esta investigación se ven efectos ligeros pero positivos, sin embargo, la cercanía podría también tener implicaciones negativas, debido a que los hábitos y estilos de vida son moderados por la proximidad de la relación, de tal manera que las parejas también se ven afectadas por el estrés u otras variables negativas de su pareja, impactando en sus comportamientos (Walker et al., 2016) pero en sentido opuesto a lo observado.

Cuando las mujeres y los hombres percibieron toque afectivo y satisfacción con el apoyo recibido de parte de su pareja en lo cotidiano, reportaron más conductas saludables de actividad física y relajación. Esto puede deberse a que el estado afectivo de los participantes mejora en función de ser acariciado por el cónyuge y que el toque es usado como una estrategia de regulación emocional interpersonal no verbal que se da de forma natural y al ser fácil de percibir, puede aumentar la capacidad de respuesta de la pareja a este estímulo, haciendo el soporte emocional más beneficioso (Debrot et al., 2013; Umberson \& Thomeer, 2020). Por otra parte, el apoyo que la pareja brinda en el día a día abarca el escenario emocional, material e instrumental y desde un enfoque funcional, los recursos intercambiados que destacan son la reciprocidad, la asistencia conductual, el dinero y pequeños favores, mientras que, en términos de salud, se distinguen la reducción del estrés y la gratificación de necesidades afiliativas (Fernández-Peña, 2005).

A pesar de que hay autores que sugieren que el matrimonio conlleva más ventajas a nivel físico para los hombres que para las mujeres-diferencia que se explica por el hecho de que ellas tienen mayor influencia positiva en el estilo de vida y los hábitos de su pareja- (Murphy, Grundy \& Kalogirou, 2007), los presentes resultados señalan que ellos van a percibir una asociación 
positiva proveniente de las variables bajo estudio, únicamente para la adopción de conductas saludables de los factores de actividad y relajación y cuidado. Sin embargo, no fue el caso para la reducción de conductas de descuido, tales como automedicarse ante la percepción de alguna molestia física, o de adicciones, como consumir tabaco y bebidas alcohólicas, resultados que difieren de los hallazgos de Jackson, Steptoe, y Wardle (2015) quienes encontraron que los cambios positivos de una persona para dejar de fumar y realizar actividad física sí se asocian de manera significativa con la influencia del estilo de vida saludable constante de su pareja

Con base en estos hallazgos es posible pensar que una variable digna de tomarse en cuenta en futuras investigaciones sería la sincronicidad en la generación de la conducta de los miembros de la pareja, ya que, como los últimos autores citados postulan, el hecho de que en un mismo momento las dos personas tomen la decisión de cambiar juntos ciertos comportamientos riesgosos para la salud, aumenta las probabilidades de mantener estos cambios positivos en el tiempo, puesto que habrá más confianza y motivación para alcanzar el objetivo juntos.

Un último hallazgo fue la correlación positiva entre la satisfacción de los hombres con respecto al apoyo de sus parejas ante la adversidad o momentos de gravedad y el mayor reporte de empleo de comportamientos de cuidado. De acuerdo con la literatura, esto se puede entender desde lo planteado por House y Umberson (1988) quienes explican, que a mayor apoyo percibido por parte de la pareja romántica, mayor regulación de pensamientos, emociones y conductas que permiten instrumentar comportamientos a favor de la salud. Por otra parte, este hallazgo también se apoya en la relación que se ha comprobado entre los comportamientos sedentarios y los correlatos psicosociales que se presentan en las relaciones de pareja, mismos que, a través del apoyo que esta brinda, configuran una de las vías más importantes para la promoción de conductas saludables de cuidado, tales como la actividad física (Pauly, et al., 2019).

A pesar de la evidencia que afirma que la adopción de hábitos de salud depende, por un lado, de que el individuo sea más propenso a adoptar determinados estilos de vida saludables si tiene con quien compartirlos (como en el caso de quienes tienen pareja), y por el otro, que la internalización de normas de conducta se facilita con un marco de referencia y modelo a seguir que ofrecen los integrantes de la familia; los resultados de esta investigación mostraron efectos bajos. Una posible explicación a este respecto se encuentra en el argumento de Noar, Chabot y Zimmerman (2008) quienes mencionan 
que, modificar conductas de riesgo que ya están instauradas en la persona resulta difícil, por lo que adquirir nuevas costumbres o comportamientos más adecuados representa un gran reto. Estos autores proponen desde el Modelo de Creencia de Salud (Becker \& Maiman, 1975) la integración con otras teorías como la Teoría de Acción Razonada (Ajzen \& Fishbein, 1980), la Teoría de la Conducta Planeada (Azjen, 1991) y el Modelo Transteórico (Prochaska \& DiClemente, 1983) varios grados de preparación para el cambio de conductas de riesgo, en el que las personas primero no tienen la intención de cambiar en el futuro previsible (precontemplación), tener la intención constante por cambiar esos riesgos por un periodo aproximado de 6 meses (contemplación), preparar el inicio del cambio al menos por 30 días (preparación), realizar el cambio (acción) y continuar haciéndolo por lo menos por 6 meses (mantenimiento), en el cual serán cruciales el balance en la toma de decisiones, la autoeficacia y los procesos de cambio y no únicamente la presencia del compañero romántico.

\section{CONCLUSIÓN}

Con los hallazgos obtenidos se puede concluir que, el hecho de estar en una relación de pareja sí crea un espacio que facilita que sus integrantes tengan mejor calidad de vida y salud, debido a la adaptación y la poderosa influencia de esta interacción sobre los hábitos y comportamientos que propician la salud de sus miembros (Holt-Lunstad, 2018), así como la influencia que se ejerce sobre las actitudes, significados y conocimientos que se tienen, favoreciendo la adopción de conductas que afectan la salud de manera positiva o negativa. Queda abierta la sugerencia de explorar en futuros estudios, cuáles otros constructos de la relación, además de las aquí evaluadas, así como variables individuales, también pueden tener influencia dentro del fenómeno estudiado y mayor peso explicativo.

Además de esta sugerencia, se resalta el hecho de que, en la aplicación de este conocimiento, se debe considerar que no existe un consenso en la forma en que es mejor realizar intervenciones destinadas a modificar conductas de riesgo o adquirir comportamientos saludables, pues tal como Guerrero y León (2010) citan, los primeros postulados a este respecto sugerían que el cambio de múltiples hábitos de vida (como dejar el cigarro, empezar a ejercitarse o mejorar la alimentación) tenían mayor éxito cuando se producían de forma secuencial y no simultáneamente, pero hallazgos más recientes demuestran la efectividad de la intervención múltiple, dejando en evidencia que en torno a este constructo poco explorado queda mucho trabajo por 
hacer, en especial al considerar las implicaciones y alcances que puede tener en términos de calidad de vida, bienestar y salud.

También se debe considerar que los estados psicológicos internos que se ven influenciados por la forma en que se convive con la pareja son la puerta de entrada que conecta a la diada con los comportamientos de salud, por lo tanto, se sugiere indagar la forma en que el estrés de un integrante para el empleo de conductas saludables puede influenciar en su cónyuge (Walker et al., 2016).

Agradecimientos: A cada uno de los participantes de la presente investigación.

Conflicto de interés: Las autoras no presentan conflicto de intereses.

Financiamiento: Investigación realizada gracias al programa UNAM-PAPIIT IN 304919 del proyecto "Factores protectores y de riesgo a la salud en parejas sanas y con enfermedad crónico-degenerativa".

\section{REFERENCIAS}

Ajzen, I. (1991). The theory of planned behavior. Organizational Behavior and Human Decision Processes, 50, 179-211. http://doi.org/10.1016/0749-5978(91)90020-T

Ajzen, I. y Fishbein, M. (1980). Understanding attitudes and predicting social behavior. New Jersey: Prentice Hall.

Alzugaray, C. \& García, F. (2015). Relaciones de pareja y bienestar psicológico. En M. Bilbao, D. Páez y J. Oyanedel [Eds.]. La felicidad de los chilenos. Estudios sobre bienestar. (pp.237-252). Santiago de Chile: RIL Editores.

Aron, A., Aron, E., \& Smollan, D. (1992). Inclusion of the other in the self-scale and structure of interpersonal closeness. Journal of Personality and Social Psychology, 63(4), 596-612. https://doi.org/10.1037/0022-3514.63.4.596

Becker, M.H. \& Maiman, L. (1975). Socio Behavioral determinants of compliance with health and medical recommendations. Medical Care, 13, 10-24. https://doi. org/10.1097/00005650-197501000-00002

Berscheid, E., Synder, M., \& Omoto, A. (1989). The Relationship Closeness Inventory: Assessing the closeness of interpersonal relationships. Journal of Personality and Social Psychology, 57(5), 792-807. https://doi.org/10.1037/0022-3514.57.5.792

Carrillo Medina, L. (2004). Relación entre estilos de comunicación, manejo de conflicto y satisfacción en la relación de pareja (Tesis de Licenciatura). Universidad de las Américas Puebla. http://catarina.udlap.mx/u_dl_a/tales/documentos/lps/carriIlo_m_l/

Chatel-Goldman, J., Congedo, M., Jutten, C., \& Schwartz, J. (2014). Touch increases autonomic coupling between romantic partners. Frontiers in Behavioral Neuroscience, 8(95), 1-5. https://doi.org/10.3389/fnbeh.2014.00095

Cortés-Vázquez, E. B., Ramírez-Guerrero, E. M., Olvera-Méndez, J. \& Arriaga-Abad, Y. J. (2009). El comportamiento de salud desde la salud: La salud como un proceso. Alternativas en Psicología, 14(20), 89-103. http://pepsic.bvsalud.org/pdf/alpsi/ v14n20/v14n20a08.pdf 
Da Silva, V. (2014). Comportamiento amoroso de pareja: Mitos y paradojas románticas: Un estudio comparativo entre Brasil y España. (Tesis Doctoral no publicada). Facultad de Psicología. Universidad Complutense de Madrid, España. https:// eprints.ucm.es/25231/1/T35350.pdf

Debrot, A. (2012). Positive Relationship Processes: Interpersonal Emotion Regulation and Well-Being in the daily life of Romantic Couples (Tesis Doctoral no publicada). Universidad Friburgo, Suiza. https://doi.org/10.13140/rg.2.1.2216.4246

Debrot, A., Schoebi, D., Perrez, M. \& Horn, A. (2013). Touch as an interpersonal emotion regulation process in couple's daily lives: The mediating role of psychological intimacy. Personality and Social Psychology Bulletin, 39(19), 1373-1385. https://doi.org/10.1177/0146167213497592

Dibble, J., Levine, T., \& Park, H. (2011). The Unidimensional Relationship Closeness Scale (URCS): Relliability and validity evidence for a new measure of relationship closeness. Psychological Assessment, 24(3), 565-572. Advance Online publication. https://doi.org/10.1037/a0026265 .

Domínguez, E. (2012). Estudio sobre satisfacción marital y variables asociadas en parejas españolas (Tesis de maestría no publicada). Universidad de Salamanca, España. https://gredos.usal.es/handle/10366/121029.

Dunkel, C. (2017). Moving research on health and close relationships forward. A Challenge and an obligation. American Psychologist, 72(6), 511-516. https://doi. org/10.1037/amp0000158.

Fernández-Peña, R. (2005). Redes sociales, apoyo social y salud. Periferia Revista de Recerca i Investigación en Antropología, 3, 1-16. https://revistes.uab.cat/ periferia/article/view/149/166.

Fife, S. \& Weeks, G. (2010). Barriers to recovering intimacy. En J. Carlson y L. Sperry [Eds.] Recovering intimacy in love relationships: A clinician's guide. (pp.157-179). New York: Routledge.

Galione, J. (2016). Relationships satisfaction and health outcomes in the context of personality. (Tesis de Licenciatura no publicada). Escuela de Artes y Ciencias. Universidad de Washington, Estados Unidos de América. https://openscholarship.wustl.edu/art_sci_etds/848/

Guerrero, L. \& León, A. (2010). Estilo de vida y salud. Educare, 14(48), 13-19. http:// www.saber.ula.ve/bitstream/handle/123456789/32226/articulo1.pdf;jsessioni$\mathrm{d}=\mathrm{F} 640 \mathrm{DDB} 6 \mathrm{~F} 225 \mathrm{A4043C46CA9F1A8CB41A}$ ?sequence $=1$

Holt-Lunstad, J. (2018). Why social relationships are important for physical health: A systems approach to understanding and modifying risk and protection. Annual Review of Psychology, 69, 439-458. https://doi.org/10.1146/annurevpsych-122216-011902.

House, J. \& Umberson, D. (1988). Social relationships and health. Science, 241(4865), 540-545. https://doi.org/10.1126/ciencia.3399889.

Jackson, S., Steptoe, A., \& Wardle, J. (2015). The influence of partner's behavior on health behavior change. JAMA Internal Medicine, 175(3), 385-392. https://doi. org/10.1001/jamainternmed.2014.7554.

Kerlinger, F., \& Lee, H. (2002). Investigación del comportamiento: Métodos de investigación en ciencias sociales. (4a Ed.) México: McGraw-Hill

Matud, P., Cabelleira, M., López, M., Marrero, R. \& Ibáñez, I. (2002). Apoyo social y salud: Un análisis de género. Salud Mental, 25(2), 32-37. http://www.inprf-cd. gob.mx/pdf/sm2502/sm250232.pdf .

Morales, D. (2016). Afecto y relación de pareja. (Tesis de Licenciatura no publicada). Facultad de Humanidades. Universidad Rafael Landívar, Guatemala. http://recursosbiblio.url.edu.gt/tesiseortiz/2016/05/42/Morales-Dulce.pdf. 
Murphy, .M, Grundy, E. \& Kalogirou, S. (2007). The increase in marital status differences in mortality up to the oldest age in seven European countries, 1990-1999. Population Studies, 61, 287-98. https://doi.org/10.1080/00324720701524466.

Noar, S., Chabot, M., \& Zimmerman, R. (2008). Applying health behavior theory to multiple behavior change: Considerations and approaches. Preventive Medicine, 46, 275-280. https://doi.org/10.1016 / j.ypmed.2007.08.001

Pauly, T., Keller, J., Knoll, N., Michalowski, V., Hohol, D.H., Ashe, M.C., Gerstorf, D., Madden, K.M., \& Hoppmann, C.A. (2019). Moving in Sync: Hourly Physical Activity and Sedentary Behavior are Synchronized in Couples. Annals of Behavioral Medicine, 54(1), 10-21. https://doi.org/10.1093/abm/kaz019.

Pozos-Gutiérrez, J., Rivera-Aragón, S., Reyes-Lagunes, I., \& López-Parra, M. (2013). Escala de felicidad en la pareja: Desarrollo y validación. Acta de Investigación Psicológica, 3(3), 1280-1297. https://doi.org/10.1016/S2007-4719(13)70967-0.

Prochaska, J.O. \& DiClemente, C.C. (1983). Stages and processes of self-change of smoking: Toward an integrative model of change. Journal of Consulting and Clinical Psychology, 51(3), 390-405. https://doi.org/10.1037//0022-0006X.51.3.390.

Ramos Salazar, L. (2015). Exploring the relationship between compassion, closeness, trust and social support in same-sex friendships. The Journal of Happiness \& Well Being, 23(1), 15-29. https://self-compassion.org/wp-content/uploads/2016/06/ Salazar-2015.pdf

Rosenblatt, P. \& Barner, J. (2006). The dance of closeness-distance in couple relationships after the death of a parent. Omega, 53(4), 277-293. https://journals. sagepub.com/doi/abs/10.2190/X1W4-M210-6522-1034.

Sánchez-Aragón, R. (1995). El amor y la cercanía en la satisfacción de pareja a través del ciclo de vida (Tesis de Maestría no publicada). Universidad Nacional Autónoma de México. http://oreon.dgbiblio.unam.mx/F/TYTIKYA7V9TX2C6AN12Q74R1G3LD2QA9BFQ17QJQ7IFQARH4J8-41557? func=short-jump\&jump=000041.

Sánchez-Aragón, R. (2018). Descubriendo la infraestructura psicosocial de la pareja romántica en México. En R. Sánchez Aragón (Ed.). Cultura y Amor en Latinoamérica: las parejas de México y Brasil (pp.21-92). México: Universidad Nacional Autónoma de México.

Sánchez-Aragón, R. (2019, junio). Apoyo de la pareja: Satisfacción, Capacidad para Recibirlo y Resiliencia en México. Revista Costarricense de Psicología. En proceso de evaluación.

Santiago-Silva, D. Z. (2020). Efectos del apego, la calidez y la empatía en la generación de comportamientos saludables de parejas heterosexuales. (Tesis de Licenciatura no publicada). Universidad Nacional Autónoma de México.

Skoyen, J. A., Rentscher, K. E., \& Butler, E. A. (2018). Relationship quality and couples' unhealthy behaviors predict body mass index in women. Journal of Social and Personal Relationships, 35(2), 224-245. https://doi. org/10.1177/0265407516680909.

Tucker, J. \& Anders, S. (2001). Social control of health in marriage. Journal of Applied Social Psychology, 31(3), 467-485. https://doi.org/10.1111/j.1559-1816.2001. tb02051.x

Umberson, D. \& Thomeer, M. B. (2020). Family matters: Research on family ties and health, 2010 to 2020. Journal of Marriage and Family, 82(1), 404-419. https:// doi.org/10.1111/jomf.12640.

Villegas Besora, M. (2006). Amor y dependencia en las relaciones de pareja. Revista de Psicoterapia, 17(68), 5-64. https://www.centroitaca.com/pdf/biblioteca/ Amor_13.pdf 
Walker, C., Lucier-Geer, M., Mancini, J., Ferrato, A., \& Ross, B. (2016). Family relational health, psychological resources and health behaviors: A dyadic study of military couples. Military Medicine, 181(2), 152.160. https://doi.org/10.7205 / MILMEDD-14-00740.

Esta obra está bajo: Creative commons attribution 4.0 international license. El beneficiario de la licencia tiene el derecho de copiar, distribuir, exhibir y representar la obra y hacer obras derivadas siempre y cuando reconozca y cite la obra de la forma especificada por el autor o el licenciante.

\section{(cc) BY}

